\title{
Editorials
}

\section{Under-representation of minority ethnic groups in research - call for action}

\section{THE IMPLICATIONS OF SUPER-DIVERSITY FOR HEALTH AND RESEARCH}

People have been coming to the UK since the beginning of recorded time. ${ }^{1}$ The latest Census shows that England and Wales have become more ethnically diverse with the majority of individuals still identifying themselves as white British (80.6\%). ${ }^{2}$

Over the past two decades, as a result of economic and political changes, migration patterns into the UK have shifted from postcolonial migration particularly referring to relatively discreet communities from the Indian sub-continent and West Indies settling in the UK, to a new type of migration with many different people arriving from many different places. This shift in pattern has resulted in a new kind of diversity which Vertovec ${ }^{3}$ has coined 'super-diversity' characterised by overlapping variables including country of origin lethnicity, language, religious tradition, regional and/ or local identities), migration experience (influenced by sex, age, education, specific social networks, economic factors), and legal status lencompassing a variety of entitlements and restrictions). Thus ethnic and cultural diversity is becoming more complex. While diversity has many benefits for the economy, science, and culture, it also presents health services and research with the challenges of meeting the needs of a population that is super-diverse in terms of their health profiles and behaviour. ${ }^{4}$

\section{THE NEED FOR ADDRESSING THE UNDER-} REPRESENTATION OF MINORITY GROUPS

It has long been recognised that despite an often greater burden of disease, ${ }^{4}$ people from minority ethnic groups are underrepresented in clinical and health research. ${ }^{5}$ This often inadvertent exclusion has serious implications for medical science by limiting validity and generalisability ${ }^{b}$ and for social justice by affecting the allocation of resources for services and research. Research involving minority ethnic groups

\author{
minority ethnic groups are willing to participate \\ in research if the study has direct relevance to them \\ and their community and if they are approached \\ with sensitivity and given clear explanations of what \\ participation involves.
}

is also relevant to the majority 'white population, as it increases understanding of the aetiology and management of long-term conditions; ${ }^{7}$ through increasing awareness of diversity and its implication for policy and practice, by improving access to and dialogue with specific communities, and highlighting the need for holistic approaches to managing illness. ${ }^{4}$

Reasons for exclusion of minority ethnic groups are complex due to subject, doctor and/or researcher, and societal factors, and it is not clear what the range of contributory factors are, what the main reasons are, and whether the real issue is one of 'planned exclusion', 'inadvertent exclusion', 'non-participation,' or a mixture of these. Indeed recent studies ${ }^{8,9}$ have highlighted that minority ethnic groups are willing to participate in research if the study has direct relevance to them and their community and if they are approached with sensitivity and given clear explanations of what participation involves. Furthermore, there is evidence from US-based research ${ }^{10}$ that suggests the reason for non-participation is less a function of negative attitudes or high levels of distrust, but rather a lack of commitment to ensuring good access to health research. Stereotypical and negative attitudes of researchers have the potential to shape decisions to recruit members of minority ethnic groups if they believe that those with poor English language skills may also lack, for instance, adequate

\section{"Research involving minority ethnic groups is also relevant to the majority 'white' population, as it increases understanding of the aetiology and management of long-term conditions.}

housing or transport, and therefore are more likely to have difficulty in keeping appointments or complying with the study protocol.11 Such attitudes will clearly limit minority ethnic representation in research. However, guidance is available to increase non-English speaker's participation. ${ }^{12}$

\section{TO MANDATE OR NOT TO MANDATE?}

The aim of health research is to determine the best strategies for preventing and treating disease and to inform health policy. To make sure that health policies serve a diverse population, it is important that all ethnic and cultural groups participate in health research. Indeed there is now a requirement for all patients to be given the opportunity to participate in research for the health and wellbeing of the population. ${ }^{13}$ Yet how this is to be achieved remains unclear.

In the US, legislation directed the National Institutes of Health $(\mathrm{NIH})$, which funds biomedical and health research, to ensure the inclusion of women and minority ethnic groups in their research. ${ }^{14}$ Since 1994 researchers are explicitly required to replicate the ethnic composition of the population they seek to study in their sample. The $\mathrm{NIH}$ also stipulated that phase III clinical trials must include subgroup analyses to assess sex and ethnic differences in treatment efficacy. It recommended that these analyses should be conducted in all clinical studies, even in cases where small sample size limits the statistical power, and despite the danger of negative consequences of a study finding a difference related to ethnicity when in reality there was none. This legislation has led to some improvements in trial participation, particularly in NIH rather than industryfunded research. ${ }^{15}$ For the increasingly super-diverse UK, this raises the question if regulation mandating inclusion is also a possible solution. 
“... engagement with communities and more personalised approaches are necessary to increase the recruitment and participation of patients from all communities, including minority ethnic communities.

The UK along with other European countries, are increasingly emphasising the benefits of making research subject populations more inclusive, but without adopting any mandatory regulations for such inclusion. The Research Governance Framework $^{16}$ requires researchers 'whenever relevant' to take account of age, disability, sex, sexual orientation, race, culture, and religion in its design, undertaking, and reporting'. However, adherence to this principle is not monitored and it is difficult to show how it has affected the recruitment of minority ethnic groups into clinical research. Alongside the Research Governance Framework, guidance on the composition of research ethics committees also emphasises the need to reflect the diversity of the population and to encourage applications for membership from groups which are under-represented, but this is also not enforced. ${ }^{17}$ We know that engagement with communities and more personalised approaches are necessary to increase the recruitment and participation of patients from all communities, including minority ethnic communities. ${ }^{8.18}$ However, these will be more resource intensive and will require funding bodies to cover associated costs. Yet to increase the evidence base for managing the patients we see in general practice, policy makers, research funders, professionals, and the public need to work together to include everyone in research.

\section{ADDRESS FOR CORRESPONDENCE}

\section{Paramjit S Gil}

Primary Care Clinical Sciences, University of

Birmingham, Birmingham, B15 2TT, UK.

\section{E-mail: p.s.gillabham.ac.uk}

\section{Sabi Redwood,}

Research Fellow in Medical Sociology, Primary Care Clinical Sciences, University of Birmingham, Birmingham.

\section{Paramjit S Gill,}

GP, Reader in Primary Care Research, Primary Care Clinical Sciences, University of Birmingham, Birmingham.

\section{Provenance}

Commissioned; not externally peer reviewed.

\section{Funding}

Sabi Redwood is funded by the National Institute for Health Research (NIHR) through the Collaborations for Leadership in Applied Health Research and Care for Birmingham and Black Country (CLAHRC$\mathrm{BBC}$ ) programme.

DOI: 10.3399/bjgp13X668456

\section{REFERENCES}

1. Commission for Racial Equality. Roots of the future. Ethnic diversity in the making of Britain. CRE: Belmont Press, 1996.

2. Office For National Statistics. Ethnicity and national identity in England and Wales 2011. Newport: ONS, 2011. www.ons.gov.uk/ons/ $\mathrm{rel} /$ census/2011-census/key-statistics-forlocal-authorities-in-england-and-wales/ rpt-ethnicity.html laccessed 24 May 2013).

3. Vertovec S. Super-diversity and its implications. Ethn Racial Stud 2007; 30(6): 1024-1054

4. Gill PS, Kai J, Bhopal RS, Wild S. Health care needs assessment: black and minority ethnic groups. In: Raftery J, Stevens A, Mant J (eds.). Health care needs assessment. The epidemiologically based needs assessment reviews. Third Series. Abingdon: Radcliffe Publishing Ltd, 2007: 227-399.

5. Hussain-Gambles M, Atkin K, Leese B. South Asian participation in clinical trials: the views of lay people and health professionals. Health Policy 2006, 77(2): 149-165.

6. Oakley $A$, Wiggins $M$, Turner $H$, et al. Including culturally diverse samples in health research: a case study of an urban trial of social support. Ethn Health 2003; 8(1): 29-39.
7. Taylor AL, Ziesche S, Yancy C, et al. Combination of isosorbide dinitrate and hydralazine in blacks with heart failure. $N$ Engl J Med 2004; 351(20): 2049-2057

8. Gill PS, Plumridge G, Khunti K, Greenfield $\mathrm{S}$. Under-representation of minority ethnic groups in cardiovascular research: a semistructured interview study. Fam Pract 2012: 30(2): 233-241.

9. Ejiogu N, Norbeck JH, Mason MA, et al. Recruitment and retention strategies for minority or poor clinical research participants: lessons from the Healthy Aging in Neighborhoods of Diversity across the Life Span study. Gerontologist 2011; 51(suppl 1): S33-45.

10. Wendler D, Kington R, Madans J, et al. Are racial and ethnic minorities less willing to participate in health research? PLoS Med 2005; 3(2): e19.

11. Lo B, Garan N. Research with ethnic and minority populations. In: Emanuel EJ, Grady C, Crouch RA, et al (eds.). The Oxford textbook of clinical research ethics. New York: Oxford University Press, 2008.

12. Plumridge G, Redwood S, Greenfield S, et al. Involving interpreters in research studies. J Health Serv Res Policy 2012; 17(3): 190-192.

13. The National Archives. Health and Social
Care Act 2012. London: The National Archives, 2012. http://www.legislation.gov. uk/ukpga/2012/7/contents laccessed 24 May 2013).

14. National Institutes of Health. NIH policy and guidelines on the inclusion of women and minorities as subjects in clinical research-amended, October 2001. http://grants. nih. gov/grants/funding/women_min/guidelines_ amended_10_2001.htm laccessed 24 May 2013)

15. Dickerson DL, Leeman RF, Mazure CM, O'Malley SS. The inclusion of women and minorities in smoking cessation clinical trials: a systematic review. Am J Addict 2009. 18(1): 21-28.

16. Department of Health. Research governance framework for health and social care. 2nd edn. London: DoH, 2005.

17. Department of Health. Governance arrangements for research ethics committees: a harmonised edition. London: DoH, 2011.

18. Rooney LK, Bhopal R, Halani L, et al. Promoting recruitment of minority ethnic groups into research: qualitative study exploring the views of South Asian people with asthma. J Public Health (Oxf) 2011; 33(4): 604-615. 